\title{
Leveraging community metadata for multimodal image ranking
}

\author{
Fabian Richter • Stefan Romberg • \\ Eva Hörster · Rainer Lienhart
}

\begin{abstract}
Searching for relevant images given a query term is an important task in nowadays large-scale community databases. The image ranking approach presented in this work represents an image collection as a graph that is built using a multimodal similarity measure based on visual features and user tags. We perform a random walk on this graph to find the most common images. Further we discuss several scalability issues of the proposed approach and show how in this framework queries can be answered fast. Experimental results validate the effectiveness of the presented algorithm.
\end{abstract}

Keywords Image ranking $\cdot$ Image retrieval $\cdot$ PageRank $\cdot$ Graph

\section{Introduction}

With the emergence and spread of digital cameras in everyday use the number of images in personal and online collections grows daily. For example, the Flickr ${ }^{\mathrm{TM}}$ photo repository now consists of more than four billion images. Such huge image databases require efficient techniques for navigating, labeling, and searching.

F. Richter ( $₫)$ · S. Romberg · E. Hörster · R. Lienhart Multimedia Computing Lab, University of Augsburg, Augsburg, Germany e-mail: richter@informatik.uni-augsburg.de

S. Romberg e-mail: romberg@informatik.uni-augsburg.de

E. Hörster e-mail: hoerster@informatik.uni-augsburg.de

R. Lienhart e-mail: lienhart@informatik.uni-augsburg.de 
In this work we focus on the goal of selecting relevant images given a query term, i.e. finding images showing content that most people associate with the query term. More specifically we aim to solve this image search problem on a large-scale community database such as Flickr where images are often associated with different types of user generated metadata, e.g. tags, date \& time, and location.

Our proposed image ranking approach has been inspired by [8] where the PageRank method [19] has been adapted to the visual domain. The PageRank approach is a method to rank webpages according to their importance. It builds a graph representing the link structure of the web. The importance of a webpage is assumed to be proportional to the number of hyperlinks pointing towards this page, i.e. the number of pages linking it. Transferring this approach to our image search task we assume that the relevance or importance of an image is proportional to the number of images showing similar content. As we consider community databases, i.e. databases with images from many different authors/photographers, this assumption is justified by the following: If an image has many close neighbors all showing the same content and being associated with similar metadata then the respective images' authors agree that this is an important shot of the respective content.

The main difficulty in such an approach is to reasonably define the similarity between two images, i.e. to determine if two images show the same content. The authors in [8] calculate the images' distance based on the number of matching local features between two images. This approach works well for landmarks or product images as in such cases typically many images exist showing the exact same object. However, when searching for object categories or scenes we cannot expect to reliably match the local image descriptors. Thus we use a more sophisticated image description based on automatic content analysis. Moreover we do not rely solely on the automatically extracted visual content description for similarity definition, but we also exploit an image description based on the available metadata. More specifically we also use an representation based on the author's tags.

However, establishing links between all pairs in a huge image collection does not scale well, as this results into a complete graph and computing similarities between images is costly. Thus we also consider scalability issues when designing the link structure of our graph. The original PageRank method introduces only a limited amount of links in the graph due to the hyperlink structure of the web. Opposing to the hyperlink structure in the web context, there exists no similar link structure between images which we can exploit in our image graph. Therefore we rely on a nearest neighbor approach and compute similarities only between images in small subsets.

Finally, computing a separate relevance score for each image and each query term is computationally inefficient. Thus, based on our scalable nearest neighbor approach, we show how to compute the relevance of an image in a query-independent fashion. We evaluate our proposed image retrieval method extensively on a realworld large-scale database in user studies.

\subsection{Related work}

There exist many works addressing the task of searching and ranking photos in (community) databases. For instance there are approaches that aim to find representative 
images of landmarks $[2,10,13,26]$. Another work aims to find iconic object images [1] using cluster centroids and identifying images with clear foreground objects.

There are image search approaches that rely on the user tags associated with the images. The main challenge is here posed by the ambiguity and subjectivity of user annotations in such community databases, making the direct application of text search approaches difficult. Therefore many approaches analyze and exploit the visual image content to improve the noisy labeling. Li et al. [12] and Liu et al. [16] both propose methods that use a visual content description to learn a tag's relevance to an image. In [12] the authors determine a tag's relevance by nearest neighbor voting. The latter work builds a graph using the tags associated with an image and performs a random walk to determine the tag relevance. The re-ranked tag lists are then used to retrieve images. We will use this approach as one baseline for our user studies conducted in Section 5.

On the other hand there are approaches that directly analyze images and rely only on a visual content description, e.g. [4, 8], where the former work uses a classifier. However, a classifier needs to be trained on (carefully) labeled data which is not available in most scenarios.

Recently there have been some works exploiting multiple modalities for image search applications. Raguram and Lazebnik [22] perform joint clustering in a space built from visual and tag descriptors to find iconic summaries of abstract concepts. Wang and Forsyth [25] retrieve object images from webpages by analyzing the surrounding text and the image itself. In [24], Schroff et al. use the surrounding text of web images for re-ranking purposes before training a SVM classifier based on visual features.

Our multimodal ranking approach has been inspired by the graph-based approach presented by Jing and Baluja [8]. Here the authors construct an image graph where vertices represent images and edge weights are proportional to the visual similarity between two images. An importance score is computed for each image and query term by performing random walk on this graph. However, in contrast to their product image search scenario, our goal is to perform retrieval of objects categories and scenes from community databases with very diverse images depicting objects in their natural context. Also, a query-dependent graph is used in [8] to compute the importance score. In this work we propose to compute a global ranking independent of any predefined query. We use multiple modalities to build our image graph, more precisely visual features and user tags. We show that our multimodal ranking method improves performance over the unimodal case. The work by Winston et al. [7] is similar to our method. However they employ a context graph in a different domain as they rank videos based on multimodal story-level similarities. Text transcriptions and visual similarity based on near-duplicate detection are used to build a graph which in turn is refined by random walk.

We also address scalability issues in our approach. In order to let our approach scale with the steadily growing size of image repositories, we exploit a nearest neighbor approach for graph construction. This idea has been motivated by [5], where the authors propose a framework for structural analysis of image databases using spectral clustering and spectral dimensionality reduction. The experimental results presented in [7] as well as the discussion in [20] prove the rationality of nearest neighbor approaches in a random walk context. 


\subsection{Contribution}

The main contributions of this paper are:

1. We present a query-by-term image ranking approach that relies on a graph where the images are linked by their similarities. We show that using similarities based on both user annotations and visual content improves results.

2. Based on this image ranking approach we propose a self-contained image retrieval method which is designed to scale well with the increasing size of image repositories. More specifically we show how to appropriately modify the link structure of the graph and how to efficiently compute the image similarities needed to build the graph.

3. In our experiments on a large-scale real-world database we demonstrate the effectiveness of our approach. Our system yields highly satisfactory retrieval results for different kinds of query terms such as object and scene categories. In addition, we evaluate an extension such that the ranking score is computed independently of the query term resulting in a very effective, scalable image search.

\subsection{Organization}

This paper is organized as follows: Section 2 describes our proposed image ranking approach. In Section 3 we discuss the implementation of the presented method in more detail and address scalability issues. An analysis of the proposed algorithm's complexity is given in Section 4 and we evaluate the approach experimentally on a large-scale image database in Section 5. Section 6 summarizes our results.

\section{Approach}

Given a large-scale collection consisting of images and their respective metadata our goal is to find images relevant to a given query term. We define a relevant image as one showing content that most people associate with the query term. For now we focus on the case where only one query term is given, however, our method can be extended to multi-term queries.

In order to perform image search given a query term, we start with a broad set of images that satisfy the query. In our implementation this set simply includes all images that have been tagged with the query term by their authors. Since this image set is derived automatically based on this simple constraint, it contains a significant number of noisy images not necessarily showing the desired image content due to the subjectivity and ambiguity of tags. Besides these images that are somehow related to the query term, we have neither additional data nor information about which images are preferred. Thus, we need to determine a score for each image indicating its relevance or importance to the current query.

Assuming that the importance of an image is proportional to the number of images showing similar content, we build a graph representing the relationships of all images in the database. Its vertices represent our images and the edges their multimodal similarity. Those multimodal similarities are based on visual and textual features. 
We then perform a random walk on this graph to determine a score for each image indicating its importance. This importance score reflects the likelihood of arriving in a certain vertex after a random walk over the given graph. We can then automatically rank the images in the former mentioned subset according to their importance score.

In the following subsections, we describe in detail how we build our similarity graph and review the random walk.

\subsection{Multimodal similarity graph}

The link structure as well as the weights associated with the edges are fundamental for the performance of our graph-based approach. Hence, the first step in building a similarity graph is to define an appropriate distance measure for comparing images.

Previous work [8] on image ranking uses only the visual content to compare images. On the other hand it has been shown in the context of query-by-example retrieval that image descriptions based on user annotations outperform a representation based on visual features. Moreover it has been shown in recent work [15, 23] that using multiple cues to find similar images boosts performance over using a single modality, either tags or visual features. Therefore we propose to use a distance measure for image comparison that combines the two modalities-user annotations and image content (see Fig. 1).

We start by computing two descriptions for an image, one for each modality. In our implementation we represent our images by automatically extracted topic distributions. Details of our image representations are discussed in Section 3. It should be noted that our ranking system is not constrained to specific representations or modalities. However we use a low-dimensional vector description of an image in its respective modality as it allows to easily compute distances between two such vector representations.

Fig. 1 Images are compared by using the similarities in two different domains, i.e. by using visual and textual features (tags)

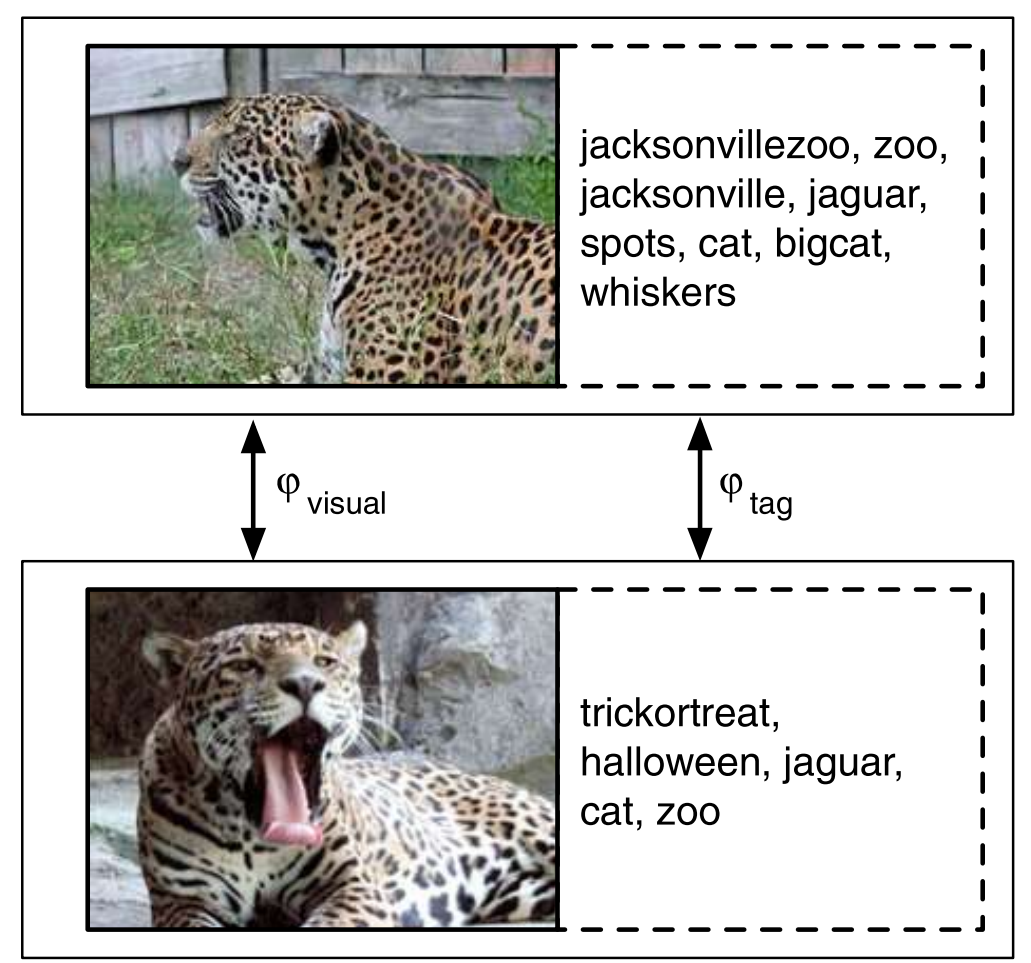


Assuming we have a vector representation of the image content for each modality $\omega$ separately, one based on text features, i.e. tags, and the other based on visual features, we define an image relevance score as

$$
\varphi_{\omega}(i, j)=\exp \left(-\frac{\left\|I_{\omega}(i)-I_{\omega}(j)\right\|_{1}}{\sigma_{\omega}}\right)
$$

where $\left\|I_{\omega}(i)-I_{\omega}(j)\right\|_{1}$ denotes in our case the $L_{1}$ distance of the representation $I_{\omega}(i)$ and $I_{\omega}(j)$ of images $i$ and $j . \sigma_{\omega}$ is a normalization constant that was set equal to the median of the pairwise $L_{1}$ distance of all images. With the equation above we obtain a relevance score between each pair of images for each modality. We then fuse both scores linearly to a combined image similarity measure $s_{i j}$ :

$$
s_{i j}=\beta \cdot \varphi_{\text {visual }}(i, j)+(1-\beta) \cdot \varphi_{\text {tag }}(i, j)
$$

where $\beta \in[0,1]$ determines the weight between visual- and tag-based features. In Section 5 we evaluate the optimal setting for $\beta$ experimentally by user studies.

Using the above defined similarity measure we are able to build our image graph. However this results in a complete graph (i.e. a graph having links between all images). Establishing, storing, and processing a fully connected graph becomes difficult when considering very large image databases, as the number of links would grow quadratically. Therefore we link each image only to its $k$-nearest neighbors, thus the number of edges grows only linearly in the number of images. Given an image we consider other images to be among the $k$-nearest neighbors if the distance between the corresponding vector representations is among the smallest $k$ distances. This is equivalent to choosing the $k$ images as neighbors which have the largest similarity according to Eq. 2.

It should be noted that due to the nearest neighbor approach, the link structure of our graph differs considerably from a complete graph. Besides its resulting sparsity, links established in the graph are not bidirectional in general. Although the used similarity defined in Eq. 2 is symmetric, the neighborhood of an image is not as it is defined by its $k$-nearest neighbors, not by the absolute distance value itself. Figure 2 visualizes the local structure of such an image graph.

As we target the search in community databases where users upload their images, it is necessary to take special care to avoid artifacts introduced by users. For instance, a single user may contribute multiple images to our image graph. Each link from one image to another represents a vote for the other image's relevance. In order to limit a user's influence, we apply the following two restrictions:

- A user may not vote for any of his own images. That is, no links between images of the same user are allowed as this would make the ranking vulnerable to manipulation by a single user.

- If an image has an incoming link from more than one image of a particular owner, the respective link weights are normalized by the number of incoming links originating from that owner's images. Alternatively we could keep only the in-link from the best matching image. 


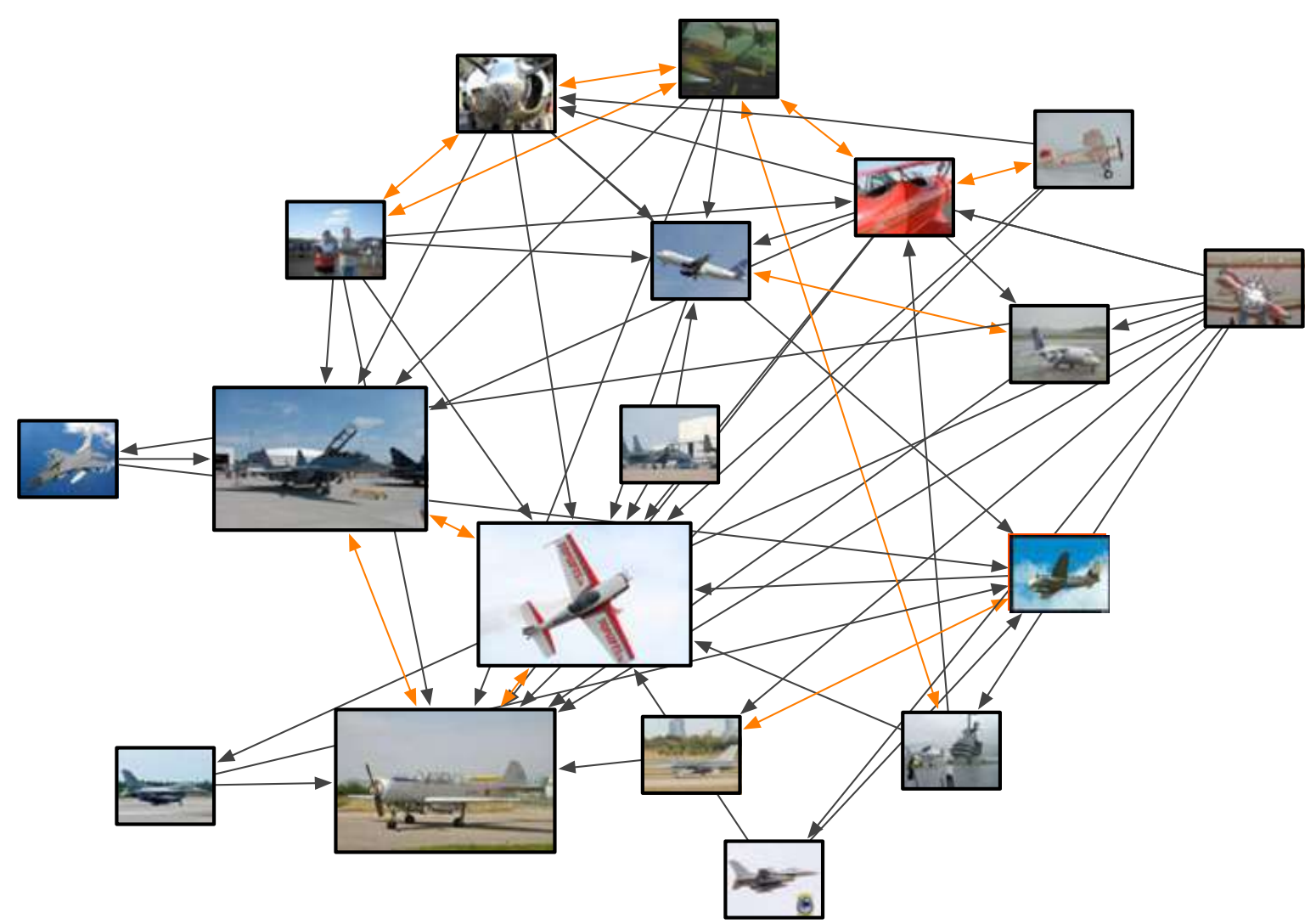

Fig. 2 Example for the link structure established in the image graph according to multimodal similarities. Note that there are both unidirectional (gray) and bidirectional links (orange) due to our nearest neighbor approach

The latter aspect is especially important since users tend to upload series of images which often show similar visual content and have the same or similar textual annotation. Thus, it is very likely that these near-duplicates share their nearest neighbors. Hence, an image voted by such a group or the group itself would be influenced overly strong by a single user.

\subsection{Random walk}

Having determined the graph representing the relationship between the images in our database we now perform a random walk on this graph. The stationary distribution of the random walk process gives us a value for each image which we use as their respective relevance scores.

Let $\mathcal{G}$ denote the image graph. Each vertex of $\mathcal{G}$ corresponds to a certain image. The edges of the graph are established as discussed in the previous subsection. The random walk then traverses the graph according to its link structure, i.e. the probability of following an edge is given by its associated weight. The final computed random walk score for a vertex corresponds to the likelihood of arriving at this vertex after random walk over $\mathcal{G}$. Thus, in order to apply the iterative random walk algorithm to $\mathcal{G}$, we construct the corresponding transition matrix $\mathbf{P} \equiv\left[p_{i j}\right]_{n \times n}$, a rowstochastic matrix describing the transition probabilities used in the random walk process, i.e. $p_{i j}=p(j \mid i)$ is the probability of arriving at vertex $j$ in one step given the current vertex $i$. 
We first compute a multimodal similarity matrix $\mathbf{M} \equiv\left[m_{i j}\right]_{n \times n}$

$$
m_{i j}= \begin{cases}s_{i j} & \text { if } j \in \mathcal{N}_{k}(i) \\ 0 & \text { otherwise }\end{cases}
$$

Each row $i$ of $\mathbf{M}$ contains exactly $k$ entries that represent weighted links to the $k$-nearest neighbors $\mathcal{N}_{k}(i)$ of image $i$. $\mathbf{P}$ is then derived by normalization:

$$
p_{i j}=\frac{m_{i j}}{\sum_{j} m_{i j}}
$$

Now let $\left[\mathbf{x}_{t}(i)\right]_{n \times 1}$ denote the so called state distribution containing all the probabilities of arriving at image (or vertex) $i$ at time instance $t$ during the random walk. Those probabilities can be computed for all future time instances by iteratively applying the transition matrix $\mathbf{P}$ :

$$
\mathbf{x}_{t+1}(j)=\alpha \sum_{i=1}^{n} \mathbf{x}_{t}(i) p_{i j}+(1-\alpha) \mathbf{v}(j),
$$

where $\mathbf{v}$ denotes a $n \times 1$ bias vector and $\alpha \in[0,1]$ is a linear fusion variable.

While the bias vector is in most cases used to ensure irreducibility ${ }^{1}$ and therefore convergence of the random walk, it also allows to assign some nodes a higher importance prior to the actual ranking procedure. Thus we examine two different bias settings in our experiments (see Section 5.3):

- A uniform bias where we assign the same bias value to all nodes.

- A non-uniform bias where the bias values correspond to a simple initial estimate of the importance of each image.

If no auxiliary knowledge is available the bias vector $\mathbf{v}$ may be initialized uniformly. Typically all entries are set to $1 / n$ with $n$ denoting the total number of images in the database. This ensures irreducibility and therefore convergence of the random walk process. On the other hand an initial estimate may improve the overall results. For instance the visual similarity computed from the image content itself could be used to obtain an initial relevance estimate for the images. However, as our experiments show (see Section 5.3), exploiting the tag lists alone provides more accurate retrieval results than using the visual similarity to compare images. Therefore we calculate a simple initial estimate based on text features derived from the images' tags and a kernel density estimator $(K D E)$ [21]. In our implementation we use this initial estimate only to set the bias values for the $L$ images having the largest values. We will determine a good choice for the parameter $L$ in our experiments (see Section 5).

Figure 3 illustrates the random walk, i.e. how the score of node $j$ is refined in iteration $t+1$ by using the scores computed in the prior iteration $t$.

\footnotetext{
${ }^{1}$ A directed graph is called irreducible, if there exists a path from each vertex to all others.
} 
Fig. 3 A partial image graph during the random walk iteration $t+1$. The score of image $j$ is refined by its neighbors $\left\{i_{1}, i_{2}, \ldots, i_{r}\right\}$ that have weighted links pointing to $j$. Their score has been computed during iteration $t$

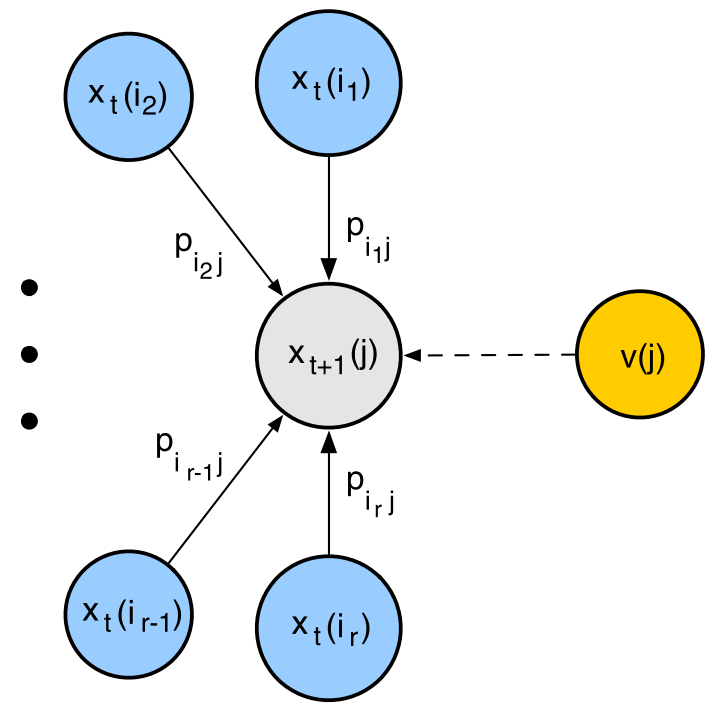

We will now show how the stationary distribution of the random walk process can be computed. Therefore we first re-write Eq. 5 more compactly:

$$
\begin{aligned}
\mathbf{x}_{t+1}^{T} & =\alpha \mathbf{x}_{t}^{T} \mathbf{P}+(1-\alpha) \overbrace{\left(\mathbf{x}_{t}^{T} \mathbf{e}\right)}^{1} \mathbf{v}^{T} \\
& =\mathbf{x}_{t}^{T}\left(\alpha \mathbf{P}+(1-\alpha) \mathbf{e v}^{T}\right) \\
& =\mathbf{x}_{t}^{T} \overline{\mathbf{P}}
\end{aligned}
$$

where $\mathbf{e}$ is a $n \times 1$ vector with all entries set to 1 . Note that, $\mathbf{v}^{T}=\left(\mathbf{x}_{t}^{T} \mathbf{e v}^{T}\right)$ as $\sum_{i} \mathbf{x}_{t}(i)=1$.

Thus, according to Eq. 6, we can express the random walk as matrix-vector multiplication, also known as the power method. With $t$ going to infinity and assuming irreducibility of $\overline{\mathbf{P}}$, the random walk converges to a (unique) stationary state distribution $\mathbf{x}_{\pi}$ :

$$
\mathbf{x}_{\pi}^{T}=\mathbf{x}_{\pi}^{T} \overline{\mathbf{P}} \Longleftrightarrow \mathbf{x}_{\pi}=\overline{\mathbf{P}}^{T} \mathbf{x}_{\pi}
$$

That is, the distribution $\mathbf{x}_{\pi}$ does not change anymore during subsequent iterations. $\mathbf{x}_{\pi}$ is then equivalent to the dominant eigenvector of matrix $\overline{\mathbf{P}}^{T}$ [9].

\subsection{Query-independent image retrieval}

We have not stated yet which images we use to build our multimodal graph. As we consider a query-by-term ranking task, two different cases are possible. Both will be experimentally evaluated in Section 5.3.

First we test a query-dependent approach where we compute the relevance scores by a random walk only for a subset of images. Typically one would pre-filter the images in the database to keep only those likely showing the query content. Thus we limit the set of candidate images used for the graph construction by keeping only those being labeled with the query term. This procedure reduces the size of the resulting graph. It may also lead to a more reliable link structure of the graph as 
image similarities may be estimated more accurately for this subset than for all images.

However, in the query-dependent case a graph needs to be built separately for every possible query. Therefore we explore a second, query-independent ranking approach. Here the relevance of an image is computed only once independent of the query term. No pre-filtering of the images is necessary and similarities are computed between all images regardless of their annotation. Thus we may experience a deterioration of the retrieval results. On the other hand, since this approach does not depend on a certain query tag, all computations can be performed offline in advance. To return the result for a certain query term $w$, we then simply need to look up the relevance score of those images of the database that are within the subset labeled with $w$.

\section{Implementation}

\subsection{Image description}

As stated above, we build our image graph using an image similarity measure based on two different modalities: The visual similarity of the image content and the textual similarity of their associated tags. To be able to apply Eq. 1, we need a fixed size vector representation of each image for both modalities. In this work we chose an image description for both the visual and textual image description based on the probabilistic Latent Semantic Analysis ( $p L S A$ ). The pLSA enables learning an abstract high-level description from the occurrence counts of low-level features like words (tags) or quantized basic visual features (commonly referred to as visual words). We derive a representation that is low-dimensional and thus, once computed, very efficient. Moreover, compared to directly using low-level image features, it describes the images' content with fewer noise by overcoming some issues, for instance polysemy and synonymy in the text features. In the visual case, Lienhart and Slaney [14] showed that comparing topic vectors yields better results than directly comparing bag-of-word histograms.

The pLSA was originally introduced by Hofmann [6] in the context of text document modeling and retrieval. The key concept of the pLSA model is to map the high-dimensional word distribution or word count vector of a document to a lower dimensional topic distribution (also called aspect vector). To achieve this, pLSA introduces a latent, i.e. unobservable, topic layer between the documents and the words. It is assumed that each document (in our case an image) consists of a mixture of multiple topics and that the occurrences of words (i.e., visual words in images or tags associated with images) stem from the topics in the respective mixture. This generative model is expressed by the following probabilistic model:

$$
p\left(d_{i}, w_{j}\right)=p\left(d_{i}\right) \sum_{K} p\left(z_{k} \mid d_{i}\right) p\left(w_{j} \mid z_{k}\right)
$$

where $p\left(d_{i}\right)$ denotes the probability of a document $d_{i}$ of the collection to be picked, $p\left(z_{k} \mid d_{i}\right)$ the probability of a topic $z_{k}$ given the current document, and $p\left(w_{j} \mid z_{k}\right)$ the probability of a visual word $w_{j}$ given a topic. $K$ denotes the number of topics. 
Although the latent topics describe the content of images, only the occurrence of words in tag lists or visual words in images can be observed in practice. To learn the pLSA model, i.e. its distributions $p\left(z_{k} \mid d_{i}\right)$ and $p\left(w_{j} \mid z_{k}\right)$, the ExpectationMaximization algorithm [3,6] is applied. Note that the learning procedure is completely unsupervised and therefore the topics themselves are defined automatically.

Once a topic mixture $p\left(z_{k} \mid d_{i}\right)$ is derived for each document $d_{i}$, a high-level representation based on the respective modality has been found. The entries of the topic vector denote to which extend an image depicts a certain topic. As we commonly choose the number of concepts in our model to be much smaller than the number of distinctive words this representation is low-dimensional. The $K$ dimensional topic mixture vector is then used to compute image similarities as in Eqs. 1 and 2.

\subsubsection{Tag features}

In order to learn the pLSA model on the image tags we need to define a finite vocabulary. We consider only the most commonly used words, i.e. our vocabulary consists of all tags in our dataset that are used by more than 100 users. Further we do not allow tags with numbers. The resulting vocabulary consisting of 2,977 distinct words was applied to derive a bag-of-words description for each image based on its associated tags. These word count vectors are then used to compute a pLSA model with 200 topics. Thus we derive a 200-dimensional tag-based image description for our text modality. We empirically chose 200 topics as a tradeoff between a lowdimensional vector and a more detailed representation.

Note that the resulting topic vector $P\left(z_{k} \mid d_{i}\right)$ computed by the pLSA is a compact representation that allows to compute similarities by simple vector operations in contrast to tag lists. Additionally the topic distribution also allows to match synonyms and homonyms when comparing images.

\subsubsection{Visual features}

To apply the pLSA model in the visual domain we consider each image as a single visual document. The pLSA can be applied directly to image tags, as image tags consist of words. However, for our visual features we need comparable elementary parts called visual words. These visual words are computed by quantizing local feature descriptors extracted from image regions.

We determine the image regions by applying dense sampling with a vertical and horizontal step size of 10 pixels across an image pyramid created with a scale factor of 1.2. SIFT descriptors [17] computed over a region of $41 \times 41$ pixels are then used to describe the local image content in a scale and orientation invariant fashion. It should be noted that any other feature detector or descriptor could be used instead. Prior to the actual feature extraction, images are scaled down to a maximum side length of 500 pixel.

Quantization is performed with a vocabulary tree [18] in order to support large vocabulary sizes. The vocabulary tree is computed by repeated k-means clustering that hierarchically partitions the feature space. Such a hierarchical approach overcomes two major problems of the traditional direct $k$-means clustering in cases where $k$ is large. Firstly, during vocabulary learning, applying the k-means algorithm 
repeatedly with small $k$ is computationally more efficient than running it only once with larger $k$. Secondly, the mapping of visual features to discrete words is very fast. In our experiments we constructed a visual vocabulary consisting of 10,000 visual words.

Once the visual vocabulary is determined we map each feature vector of each image to its closest visual word. Then, we derive a bag-of-visual words representation by counting the occurrences of each visual word in the respective image. Note that this image content description does not preserve any geometric relationship between the occurrences of the visual words.

The word count vectors are then used to compute the pLSA model. Similar to the pLSA on tags we used 200 topics leading to a 200-dimensional description of each image's content based on visual features.

\subsection{Nearest neighbor search}

As the random walk requires to find the $k$-nearest neighbors of each image, a naive implementation would result in computing similarities between all image pairs and sorting those similarities for each image. This would result in at least $O\left(n^{2}\right)$ comparisons which certainly limits the scalability of our approach, especially in the query-independent case (see Section 2.3).

Therefore we propose an approach that hierarchically partitions the topic space. The nearest neighbor search of an image is then limited to such a subspace, i.e. to a subset of images in our database. This way we reduce the number of image comparisons required for the graph construction to a linear amount depending on the cluster sizes.

To determine such a partition we cluster the images' representations, in our case their topic vectors, hierarchically by applying the k-means algorithm recursively. Each of the resulting cascaded subspaces is represented by its respective cluster centroid, i.e. the mean vector over all representations in the cluster. The resulting tree allows searching the topic space efficiently for the nearest cluster for a given image description. This is done by propagating down the tree, i.e. comparing the descriptor vector to the centroids at each level and choosing the closest. Once the nearest cluster is determined the image only needs to be compared to all members of that cluster (see Fig. 4).

The more clusters emerge from the hierarchical clustering, the faster the $k$-nearest neighbor search will be as clusters tend to be smaller and thus fewer vectors need to be compared. On the other hand, a larger number of subsets may lead to a performance degradation due to the introduction of inaccuracies at subspace borders. For our dataset consisting of roughly 260,000 images (see Section 5.1) we choose an hierarchical clustering into two clusters on each level, thus we construct a binary tree. As long as a cluster consists of more than 25,000 associated vectors it is further recursively sub-divided. This procedure results into 15 subspaces/image subsets with cluster sizes ranging from 10,740 to 23,889 vectors. We use the multimodal similarity measure as defined in Eq. 2 as our distance measure for computing the clusters.

As this $k$-nearest neighbor search is an approximation, we also empirically measured the intersection of the nearest neighbors computed with and without approximation in order to get an idea of the scale of the inaccuracies we have introduced by the approximation. Surprisingly only $34 \%$ identical images could be found 
Fig. 4 The hierarchically partitioned topic space. For a given query vector (blue dot) the nearest cluster is found and then the nearest neighbors are computed by comparing the vectors belonging to the same cluster (red dots)

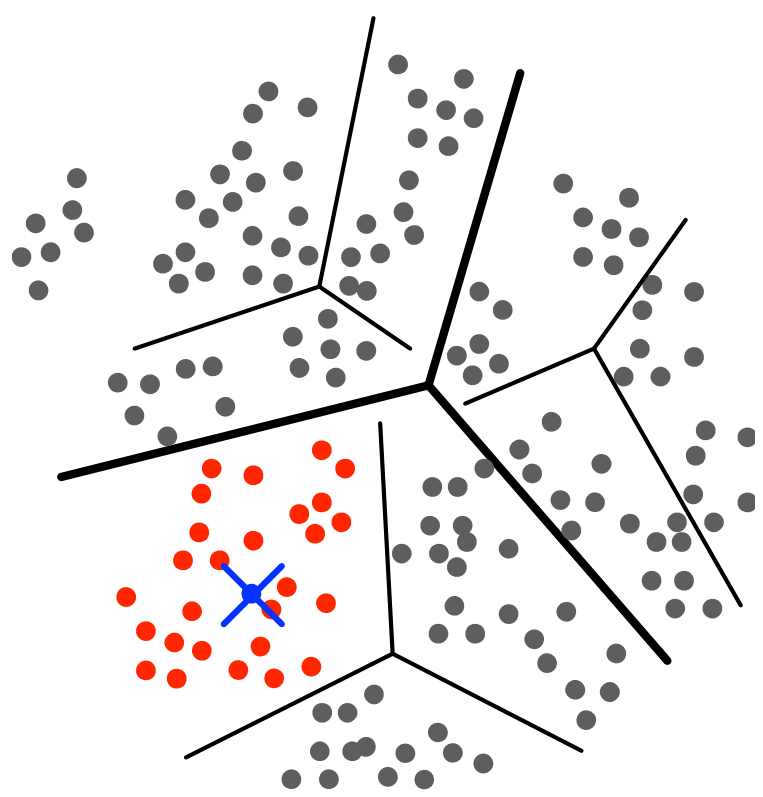

in our experiments. However, this has no negative impact on the overall performance of our query-independent system as shown in Section 5.3.

\subsection{Dynamic graph update}

When exploring our query-independent approach, retrieval results are determined by a simple lookup of each image's probability from the stationary state distribution $\mathbf{x}_{\pi}^{T}$ resulting from a random walk over the multimodal similarity graph build from all images in the current dataset. However, social online community databases are highly dynamic and images may be added and deleted frequently.

Thus updating $\mathbf{x}_{\pi}^{T}$ and/or computing the relevance scores for novel images becomes an important aspect in practical situations. In this work we do not concentrate on this issue, however we will give a short overview of possible solutions in the following paragraphs.

A naive approach to obtain a stationary state distribution $\widetilde{\mathbf{x}}_{\pi}^{T}$ for the modified graph is to re-run the entire ranking algorithm. This basically requires to re-construct the multimodal similarity graph for the modified database and to perform a random walk from scratch. Even though this approach yields the optimal state distribution according to our algorithm, the problem size makes this procedure prohibitively expensive in practice.

On the other hand there exist research works addressing the issue of obtaining the updated state distribution vector $\widetilde{\mathbf{x}}_{\pi}^{T}$. One example is the iterative aggregation updating algorithm proposed in [11]. The key idea of this algorithm is to partition the image set $S$, containing all images including the newly added ones, into two subsets, i.e. $S=G \cup \bar{G}$. Thereby $G$ contains all images that are likely to be affected by the database update, and $\bar{G}$ contains all other images. Hence, images that have been either added/deleted, have an updated tag list or obtain new nearest neighbors after the update are contained in $G$. The algorithm then computes an approximate stationary state distribution for a smaller graph that comprises all images from $G$ as 
well as a node representing all images of $\bar{G}$. It should be noted that the stationary distribution $\mathbf{x}_{\pi}^{T}$ for the images in $\bar{G}$ is unaffected by the computations.

Such an algorithm allows to efficiently compute an approximate updated stationary state distribution by considering the prior result $\mathbf{x}_{\pi}^{T}$. Thereby it accounts for both, the element-updating problem (modified link structure, constant number of nodes) as well as the state-updating problem (modified nodes, i.e. added/deleted nodes).

Moreover, the algorithm in [11] can be employed in conjunction with the extrapolation technique introduced in [9].

\section{Computational complexity}

In order to show the scalability of our query-independent approach we compare its complexity to the complexity of a naive approach which uses a complete graph, i.e. each pair of images is linked within the multimodal similarity graph. For our analysis we only consider the query-independent case which is more relevant in practice.

In a naive approach the construction of the graph requires $n^{2}$ image comparisons, where $n$ is the number of images in the database. In addition, each iteration of the power method requires $n^{2}$ floating point multiplications. As the number of necessary iterations $M$ is small in practice and considered independent of $n$, the total number of required operations is bounded by $n^{2}+M n^{2}=n^{2}(1+M)$, such that the overall complexity is $\mathcal{O}\left(n^{2}\right)$.

Now we analyze the complexity of the query-independent nearest neighbor approach using hierarchical $k$-means. As with the naive approach we first construct a similarity graph and then perform the power method. To efficiently find the nearest neighbors of an image during graph construction, we hierarchically partition our feature space and look for similar images only in such a subspace, i.e. in a subset of images (see Section 3.2). Thus, to determine the overall complexity we have to take into account the costs of hierarchical clustering used to partition the image space.

\subsection{Graph construction}

Performing a hierarchical $k$-means clustering using $k=2$ results in a binary tree. Leaves of the tree correspond to subsets of images which are then used for the nearest neighbor search and inner nodes represent clusters that are further sub-divided. During construction a cluster $C_{i}$ is further partitioned if it contains more than $W$ images. To partition the cluster $C_{i}$ we perform $k$-means clustering on $q$ random samples from within $C_{i}$. Let this $k$-means clustering require $Q$ operations known to be only dependent on $q$ and $k$.

Then we can obtain a realistic upper bound for the required number of clusterings by assuming that each $k$-means clustering sub-divides the images among two clusters by a constant factor $p$ and $(1-p)$, respectively. Without loss of generality, we may assume that given a $p, p \in(0,1)$, the $\left|C_{i}\right|$ images in cluster $C_{i}$ are divided proportionally into a smaller subset containing $p \cdot\left|C_{i}\right|$ images and a larger subset consisting of $(1-p) \cdot\left|C_{i}\right|$ images. A visualization for the resulting binary tree is depicted in Fig. 5. In this scenario, we may determine an upper bound for the required number of times $r$ running the $k$-means algorithm by $r \leq-(1 / \log (1-p)) \log n$. In this case, 
Fig. 5 Binary tree resulting from a non-optimal hierarchical clustering. Inner nodes (grey) are further sub-divided such that each leaf (blue nodes) contains only a specified maximum number of images

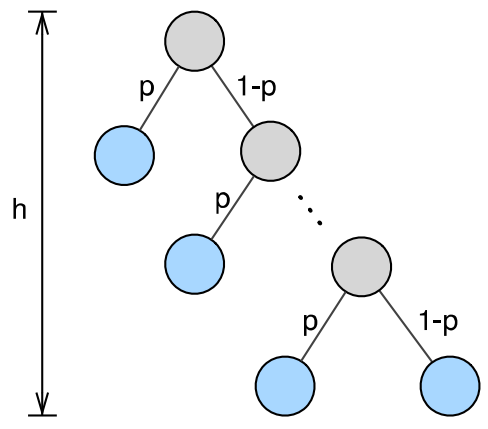

each clustering corresponds to one additional level in the resulting binary tree. This directly lets us estimate the tree height $h=-(1 / \log (1-p)) \log n$. Thus the total number of clustering operations to build the tree is bounded by $Q \cdot h$.

We also need to assign every image in the database to exactly one cluster, i.e. one leaf of the tree. This requires $n \cdot 2 h$ vector comparisons to traverse the tree down to the best-matching leaf. It should be noted that in practice this assignment is performed simultaneously during tree construction, as it is needed to determine the exact cluster size $\left|C_{i}\right|$.

Once all images are assigned to clusters, determining the links for a single image requires only one lookup of the image's cluster and at most $W$ image comparisons. Additionally, we need $W \log W$ operations to sort the similarities and thus to obtain the nearest neighbors. Therefore, the graph construction requires at most $n(W+W \log W)$ operations in total whereby the constant $W$ does not depend on $n$.

\subsection{Power method}

In this setting the transition matrix $\mathbf{P}$ contains exactly $n \cdot k$ positive entries that require $n \cdot k$ floating point multiplications within each of the $M$ iterations of the power method. Hence, in total $M \cdot n \cdot k$ operations are needed.

Summarizing all steps, the overall boundary for the number of operations is given by $Q \cdot h+n \cdot 2 h+n(W+W \log W)+M \cdot n \cdot k$. With $h=-(1 / \log (1-p)) \log n$ the overall theoretical complexity is $\mathcal{O}(n \log n)$ compared to $\mathcal{O}\left(n^{2}\right)$ of the naive approach. It should be emphasized that in practice the complexity grows only linearly in $n$ as $W>>\log n$ holds even for very large databases. Even though the tree-based nearest neighbor approach introduces computational overhead it enables us to apply it to large databases at all.

\section{Evaluation}

\subsection{Datasets}

We evaluate our approach experimentally on two datasets. The first dataset consists of 261,901 Flickr images. To derive this dataset we downloaded up to 10,000 images from each of the following 28 categories: aircraft, beach, bicycle(s), bird(s), boat, bottle(s), building, bus(es), car(s), cat(s), chair(s), city, coast, cow(s), desert, dog, 
Fig. 6 Some randomly picked images from the image set consisting of 28 categories

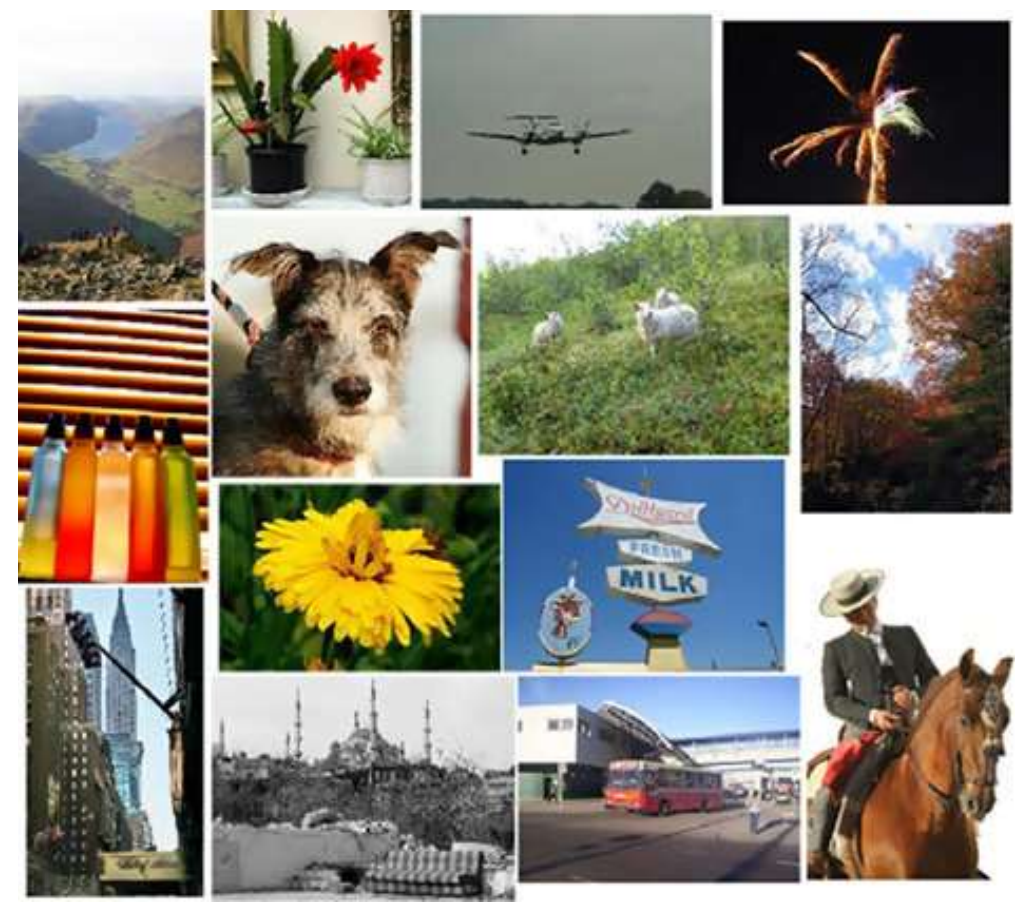

forest, horse(s), motorcycle(s), mountain(s), people, potted plant, sheep, sofa, street(s), table(s), tree(s), tv. As we downloaded images according to their user tags, images with multiple tags can be part of multiple categories. Note that the dataset contains objects as well as scene categories. Some example images from the dataset can be seen in Fig. 6.

In order to show the scalability of our approach, we also report results on an even larger second dataset. Here we collected 1,176,020 Flickr images from 56 different categories including the 28 mentioned above: aircraft, beach, bicycle(s), bird(s), boat, bottle(s), building, bus(es), car(s), cat(s), chair(s), city, coast, cow(s), desert, dog, forest, horse(s), motorcycle(s), mountain(s), people, potted plant, sheep, sofa, street(s), table(s), tree(s), tv, ship, hot air balloon, tractor, flowers, trains, butterfly, carnival, sunset, portrait, golden gate bridge, tower bridge, colosseum, cn tower, statue of liberty, pyramids, sydney opera, eiffel tower, louvre, pisa tower, arc de triomphe, snow boarding, sailing/sailboat, rock climbing, polo, baseball, soccer, christmas, wedding. Note that some categories have up to 30,000 images.

Both databases have not been cleaned or post-processed. A manual statistical analysis has shown that on average about $40 \%$ of the images are mislabeled with tags that do not describe the depicted image content (see Section 5.3).

We have also stored the metadata associated with the images including their tags. We counted 136,371 unique tags in our first collection, leading to a vocabulary of size 2977 after filtering (see Section 3.1). For the second dataset we counted 396,913 unique tags resulting in a vocabulary of size 3080 after filtering.

\subsection{Methodology}

As no ground truth is available for both large-scale image databases, test users are required to rate the performance of our system. Therefore we evaluate our ranking 
system and the different parameter settings by conducting several user studies. For each category of an image database the most frequently occurring tag is selected as the query term resulting into 28 query terms for the smaller first dataset. As the second dataset is a superset of the first collection the queries are identically used to evaluate the second dataset. Then we ask 10 participants to judge the top 19 results for every query term as "relevant", "somewhat relevant" or "not relevant" to the query.

We use the following scoring to get a quantitative performance measure: An image considered being relevant receives 1 point, an image considered as somewhat relevant receives 0.5 points. All other images get 0 points. A mean score is calculated for each user; the mean over all users' means yields the final score of the parameter configuration/system being evaluated.

\subsection{Experiments}

In our first experiments we examine the influence of several parameters in our image ranking approach using the query-dependent setting. Then we compare our querydependent image ranking method to three baselines. Finally we compare the result of the query-dependent image ranking method to the proposed query-independent algorithm. We perform all of our evaluations on the smaller dataset of 261,901 images and finally validate the performance of our query-independent approach on the larger collection of 1,176,020 images (see Section 5.1).

First, we determine the optimal weight $\beta$ for the two modalities in our multimodal similarity measure (see Section 2.1). Therefore we vary $\beta$ and keep all other parameters fixed: We chose $k=250$ nearest neighbors and a non-uniform bias to weight the top 500 positive entries for building the graph. Figure 7 shows the results of our user studies. As can be seen, the best results are obtained for $\beta=0.2$, i.e. the text modality receives a four times larger weight than the visual modality. Comparing images by their associated tags seems to be slightly more reliable than comparing them by visual content. However, fusing both similarities as proposed improves the results over using only one single modality to measure similarity. Hence, we fix $\beta$ to 0.2 for the following experiments.

Fig. 7 The results shown for different weights $\beta$ of the visual and textual modalities

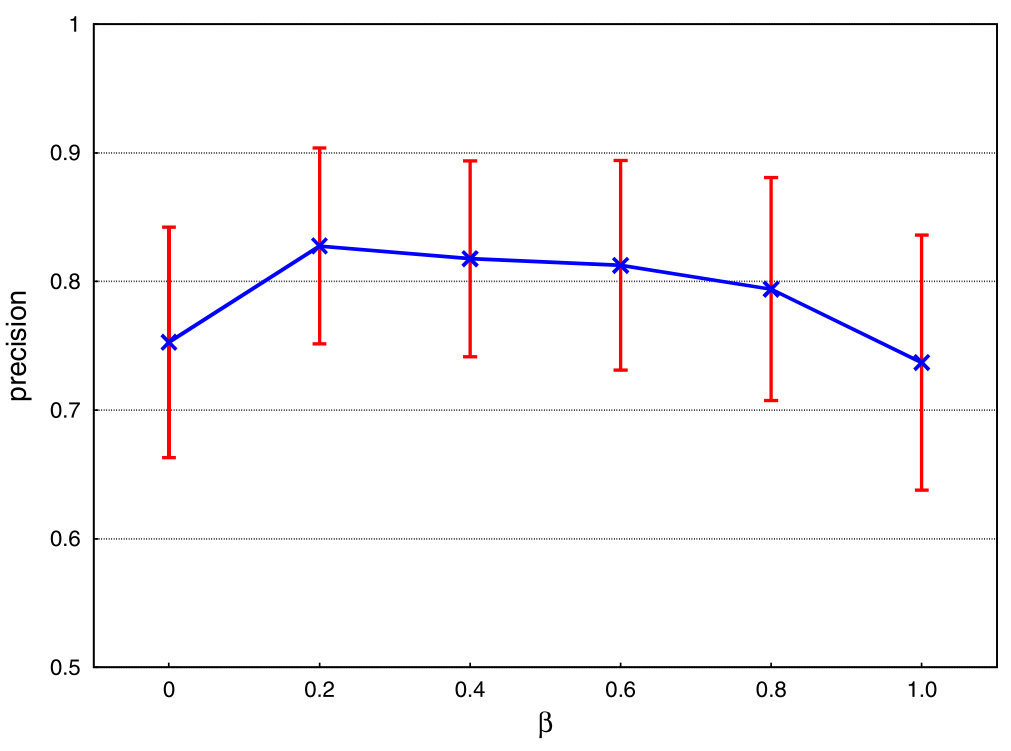


Fig. 8 The results shown for a varying number of $k$-nearest neighbors of each node

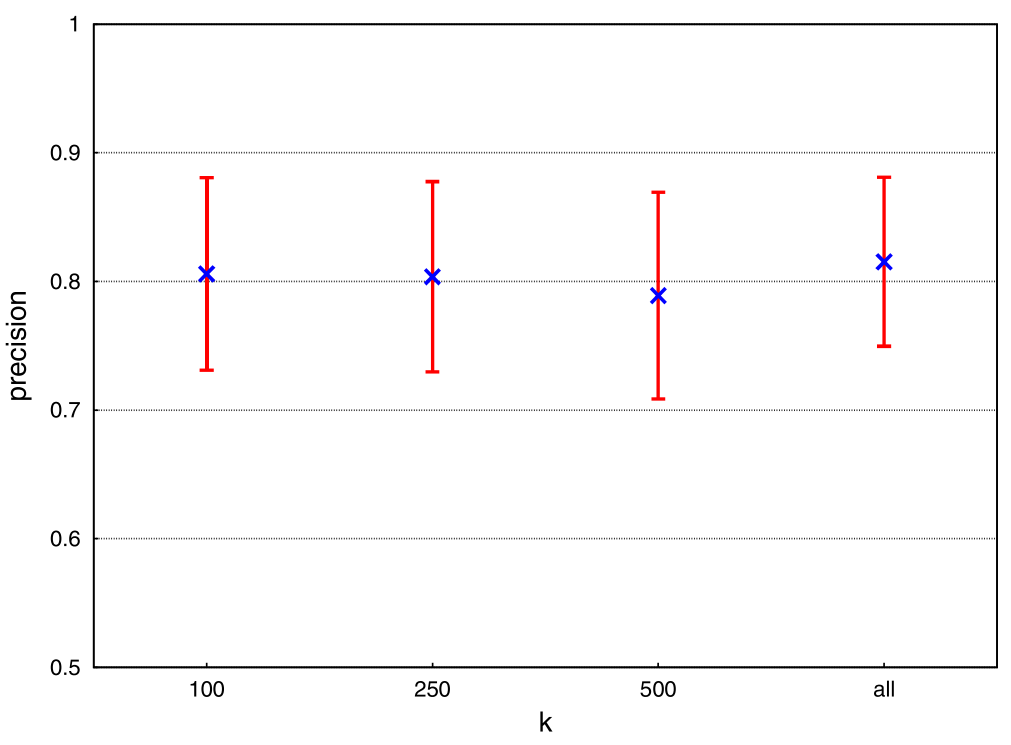

In our second experiment we vary the number of nearest neighbors $k$ used to establish the link structure in our image graph. Since we assume larger values of $k$ to introduce unreliable links to the graph, we choose $k \in\{100,250,500\}$ and compare to the results from a fully connected graph. When changing $k$ we need to adapt the bias in order to weight the nodes with the same magnitude. To avoid this issue, we chose a uniform bias to obtain comparable results. As can be seen from Fig. 8 our nearest neighbor approach yields similar results compared to an approach with a fully connected graph. However building a fully connected graph does not allow very large image sets as discussed in Section 4.

In our third experiment we evaluate the impact of the bias. Therefore we compare a uniform bias to non-uniform biases as described in Section 2.2. For the non-uniform bias we examine two different values for the parameter $L, L=500$ and $L=1,000$, indicating the number of images biased with their respective initial estimates. The other parameters, $k=250$ and $\beta=0.2$, have been chosen according to the results

Fig. 9 The results for different types of biases

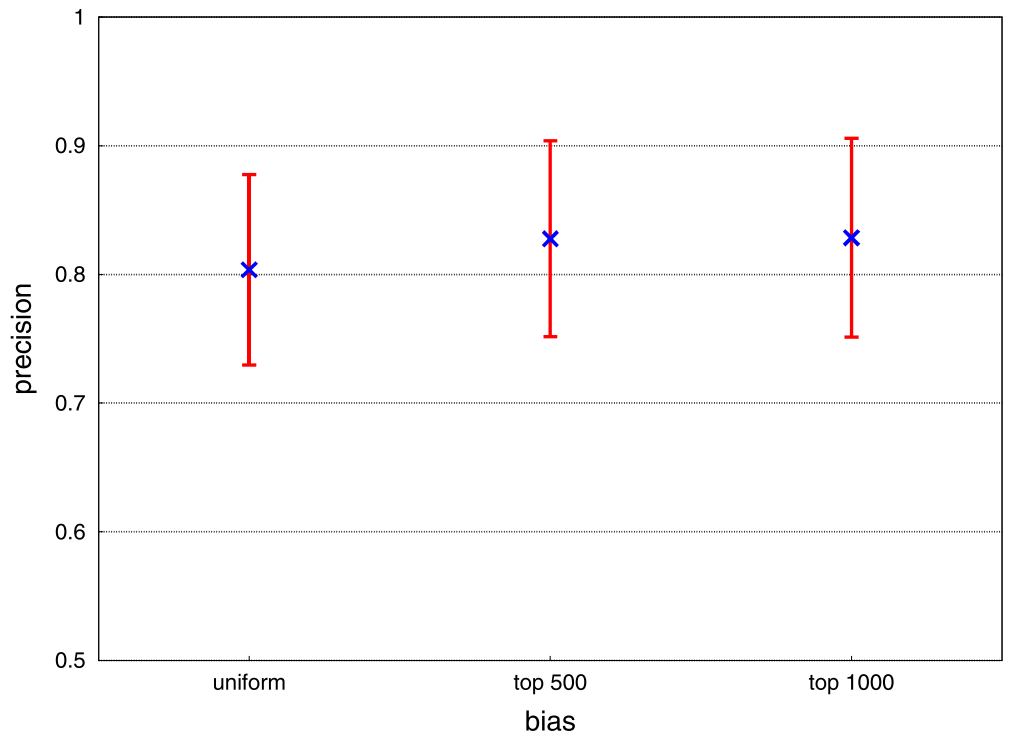


Fig. 10 Comparison between KDE-based retrieval and our query-dependent approach

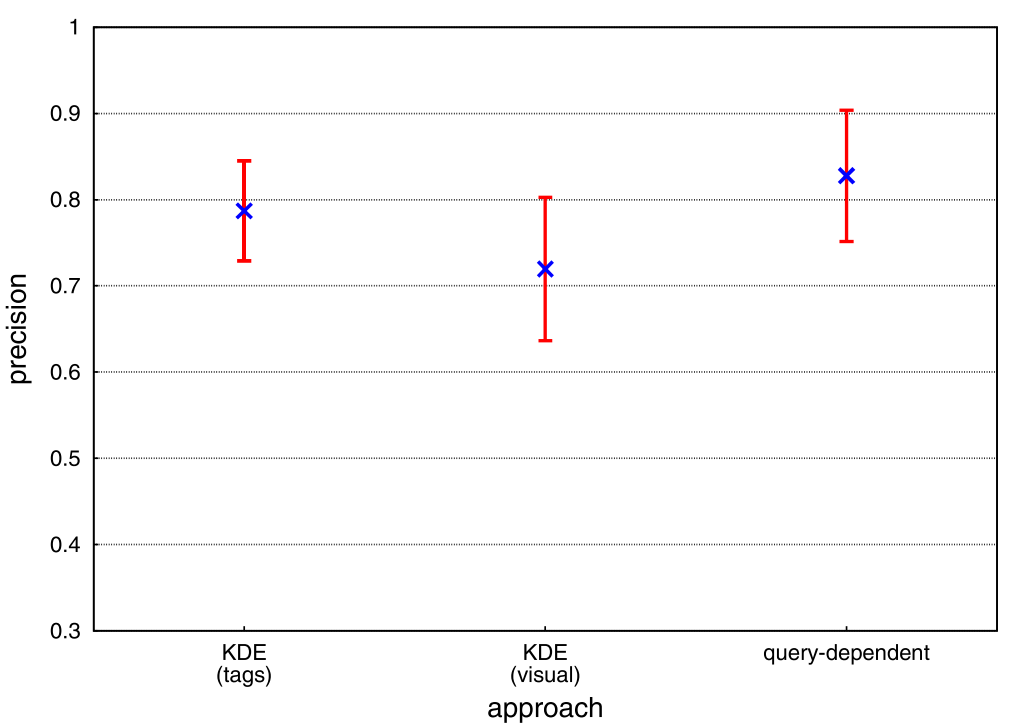

of the previous two experiments. As illustrated in Fig. 9 we obtain the highest score for a non-uniform bias where either the top 500 or top 1,000 most important images have been biased. However, the influence of the bias is small, especially since we have empirically chosen $\alpha=0.9$ (see Section 2.2).

Having determined the optimal parameter setting, we now examine the influence of the random walk. As described before, to obtain appropriate values for the nonuniform bias, we derive an initial estimate of the importance of each image based on text features and a kernel density estimator (KDE). Similarly, it could be computed based on the visual image representation. Such an importance estimate can be used to directly rank all images associated with a query term and thus to find relevant images. In Fig. 10 we compare the retrieval results obtained by using our initial estimate directly to the results after the random walk algorithm has been applied. As can be seen, the simple KDE-based method performs already well but an additional performance improvement can be obtained by the random walk. Moreover we observe

Fig. 11 A performance comparison of different image ranking approaches

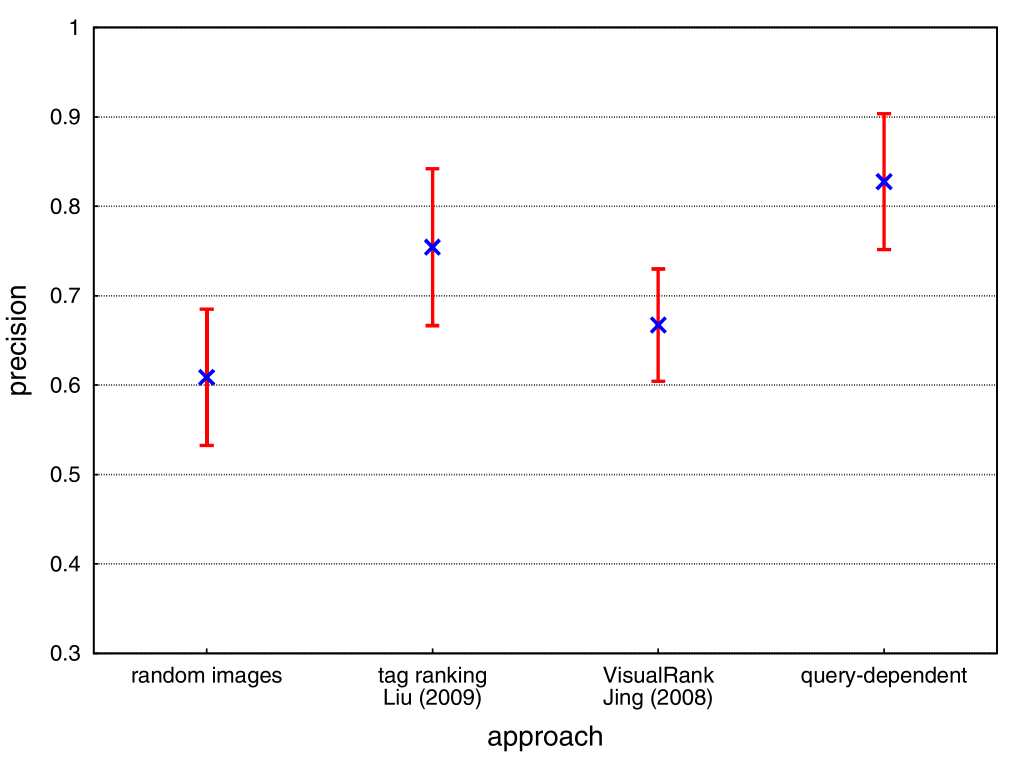


that the textual features outperform the visual image representation thus validating our choice for computing the bias values.

We now compare our query-dependent approach to three different baselines. As a worst-case baseline we draw random images from each of the 28 query term categories. This baseline reflects the average relevance of images within the tag categories included in our user studies. We also compare our system to state-of-theart approaches described in [16] and [8]. In [16] the tags associated with an image are first re-ranked using both, image content and associated tags. Given a query tag $w$, the images are then ranked according to a relevance score $r(x)$ purely relying on the
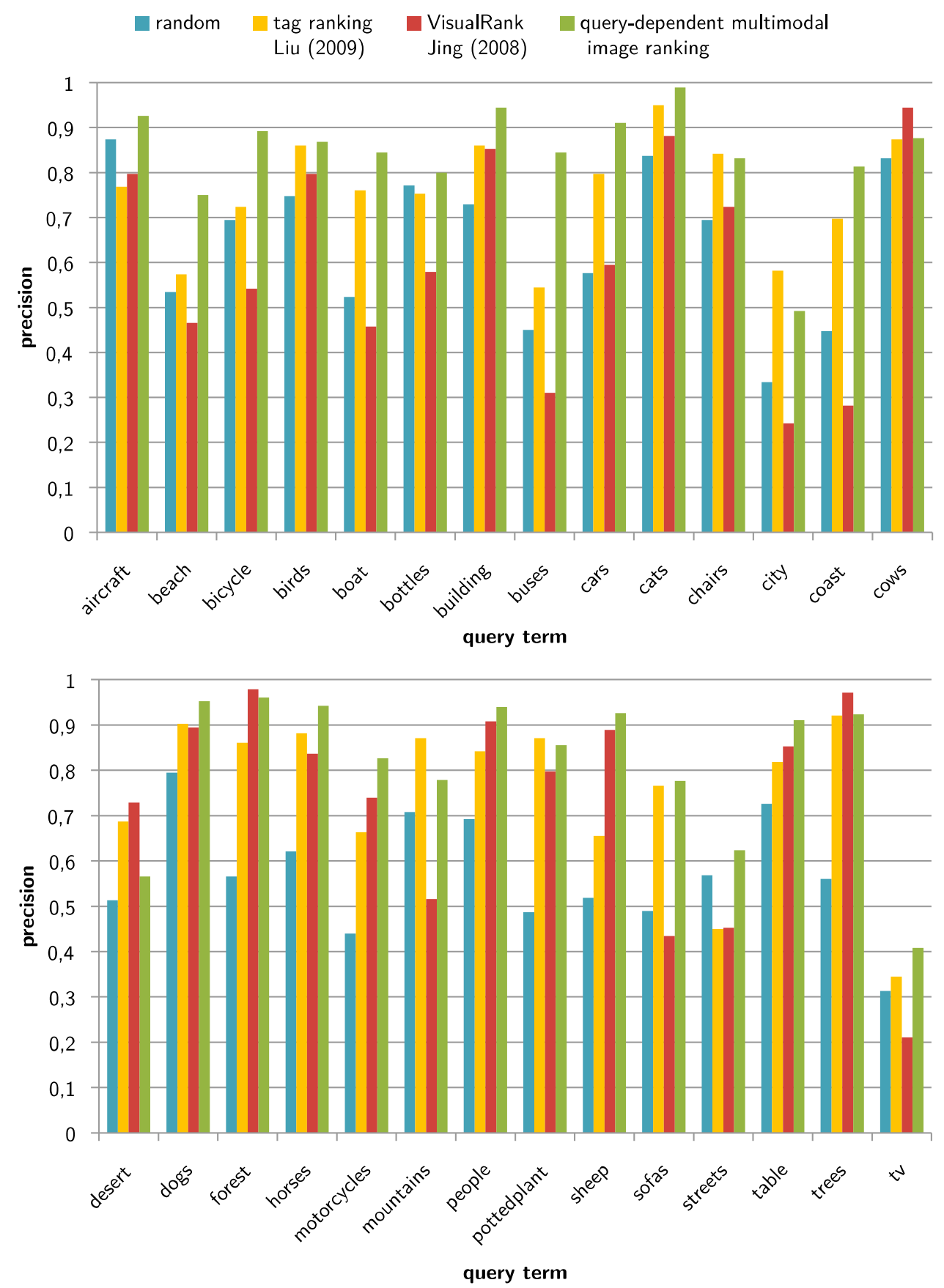

Fig. 12 Comparison between our query-dependent approach and both baselines per query term 
re-ranked tag list:

$$
r(x)=-\tau(w)+1 /|x|,
$$

where $\tau(w)$ denotes the ranked position of $w$ in the tag list of length $|x|$ associated with image $x$. The approach described in [8] applies the PageRank algorithm to an image graph built only using visual similarities between images. The similarity between two images is defined as the number of matching SIFT features. The results of our user study are depicted in Fig. 11. In Fig. 12 we also show the scores for each query term separately. One can see that our proposed multimodal query-dependent image ranking outperforms all three baselines in average and in most categories. Note that, while the tag ranking approach performs well, we consider it hardly usable in practice, as it has a strong focus on very short tag lists.

In our last experiment we evaluate the proposed query-independent approach. As can be seen in Fig. 8, the query-dependent approach performs best for the complete graph in which a link between each image pair is established. Besides that building a complete graph is not feasible in practice for the query-independent approach, a global link structure would introduce many noisy links due to the large number of unrelated images. Such links between unrelated images provide only little or no information about their similarity as the distance between their representations is not quantitatively meaningful. Therefore we empirically set the number of neighbors $k$ to 500 to build the graph. As described in Section 3.2 we performed the $k$-nearest neighbor search by utilizing the hierarchically clustered topic space. The results show that the query-independent approach allows to compute the relevant images in a scalable way with only a minor loss of precision. Moreover, as can be seen in Fig. 13, the query-independent approach unexpectedly performs better when the tree is used to approximate the $k$-nearest neighbors than determining the neighbors by direct computation. This might be due to the clustering as it divides the vector space of our image representation largely based on the tags of our images. Therefore irrelevant neighbors might be excluded as in the query-dependent case before the absolute distance between images is taken into account. To show the scalability of our query-independent approach we further test on the larger data set containing more

Fig. 13 The query-dependent approach compared to the query-independent approach

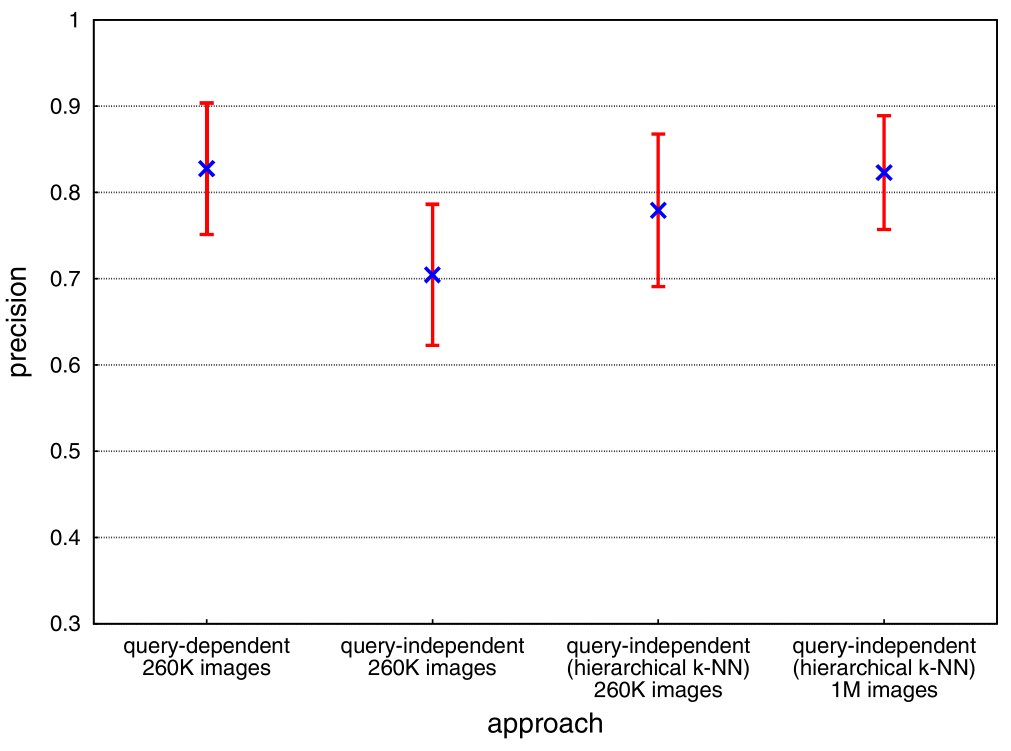


than 1.1 million images. We again use a hierarchically clustered topic space to determine the $k=500$ nearest neighbors in order to build the image graph. The improvement in performance on the larger database compared to the smaller image collection may be explained by the larger number of images per tag category. This may lead to a more accurate similarity graph as the nearest neighbors of an image can be determined more precisely due to the larger number of candidate images.
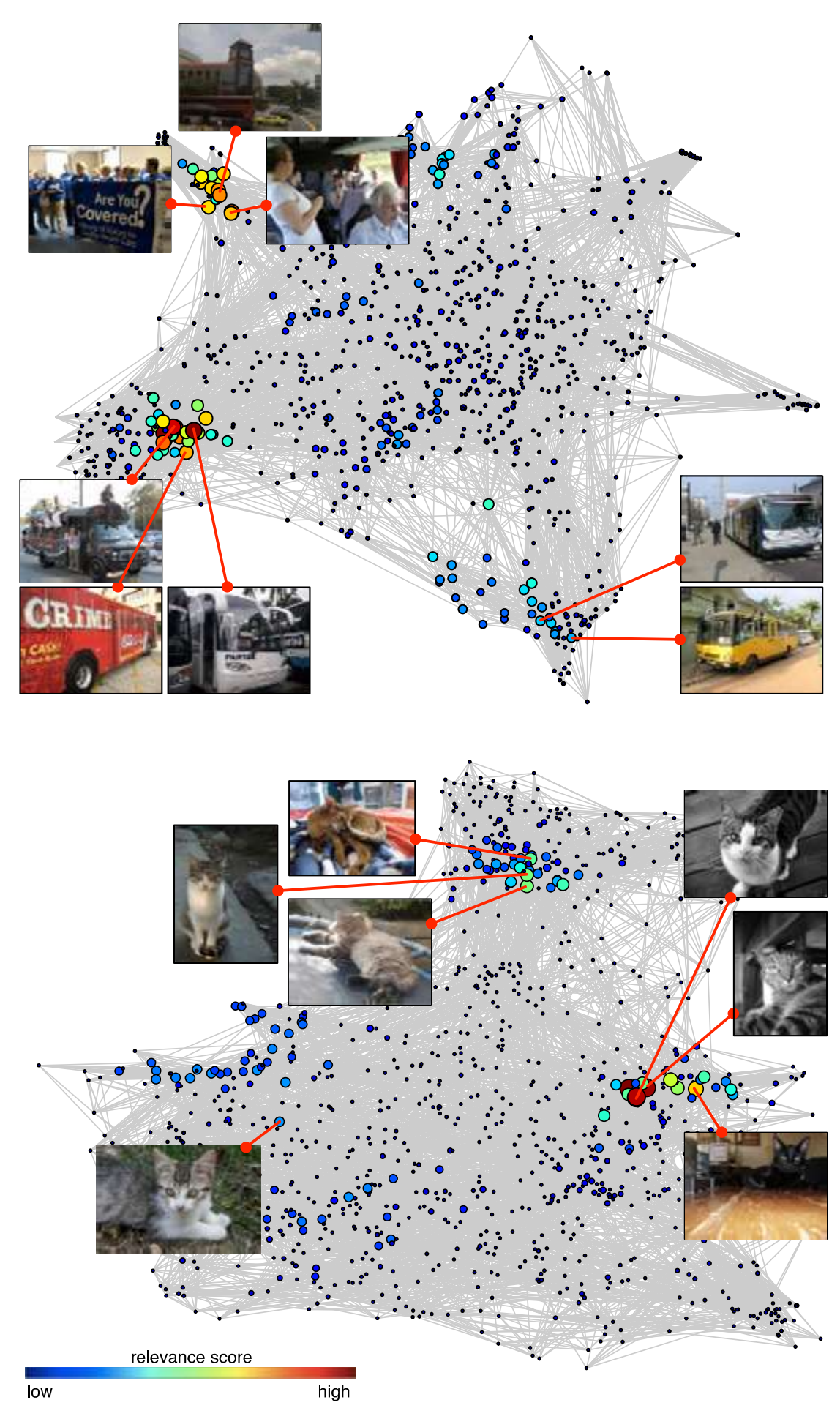

Fig. 14 Example graph for the queries bus (top) and cat (bottom) 
Fig. 15 Top 16

(query-dependent) retrieval results for query term building

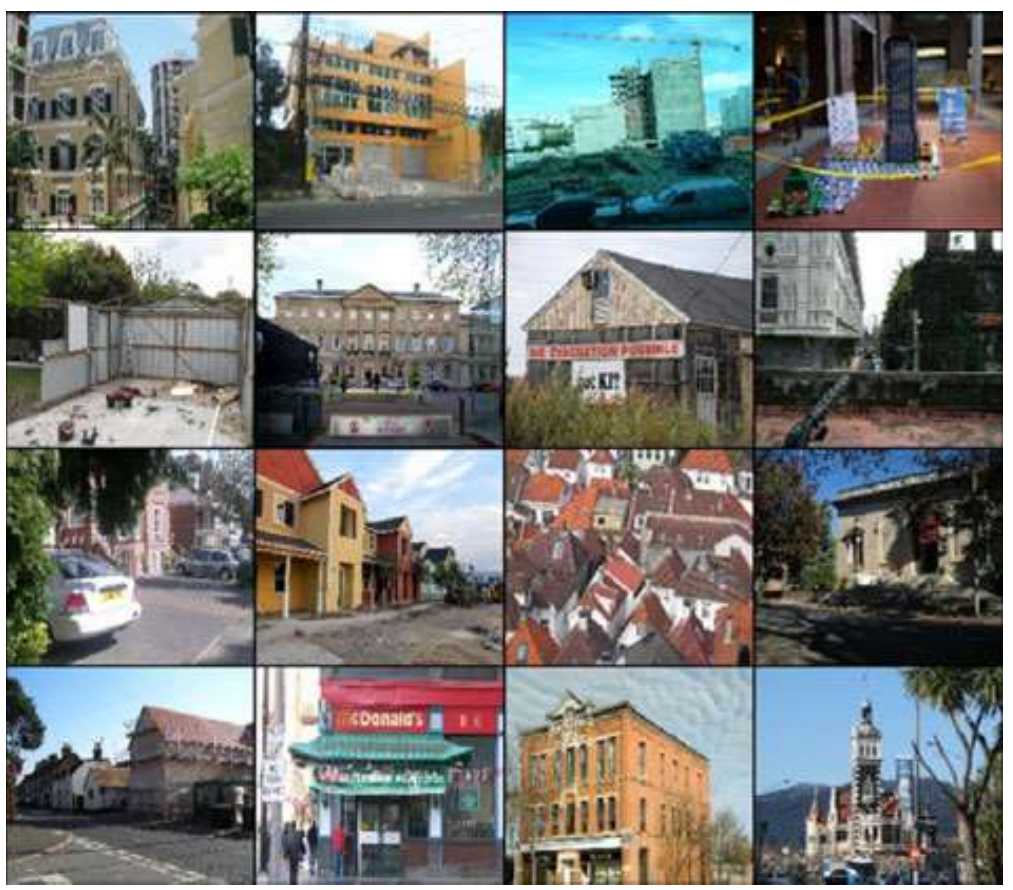

Next, we plot in Fig. 14 two result graphs to show some examples of our algorithm. We randomly selected 1.000 images for the two query terms bus and cat and applied our proposed algorithm to determine the relevance score for each image. To build the graph we set the number of nearest neighbors $k$ to 10. Each graph has then been layouted by a spring layout algorithm that groups images by their similarity. The size and color of the nodes in the graphs indicate the relevance of the according image.

Finally, we show that for both the query-dependent and query-independent approach we are able to retrieve relevant and at the same time diverse result images for different query terms such as objects, sceneries and animals. Figures 15 and 16

Fig. 16 Top 16

(query-dependent) retrieval results for query term coast

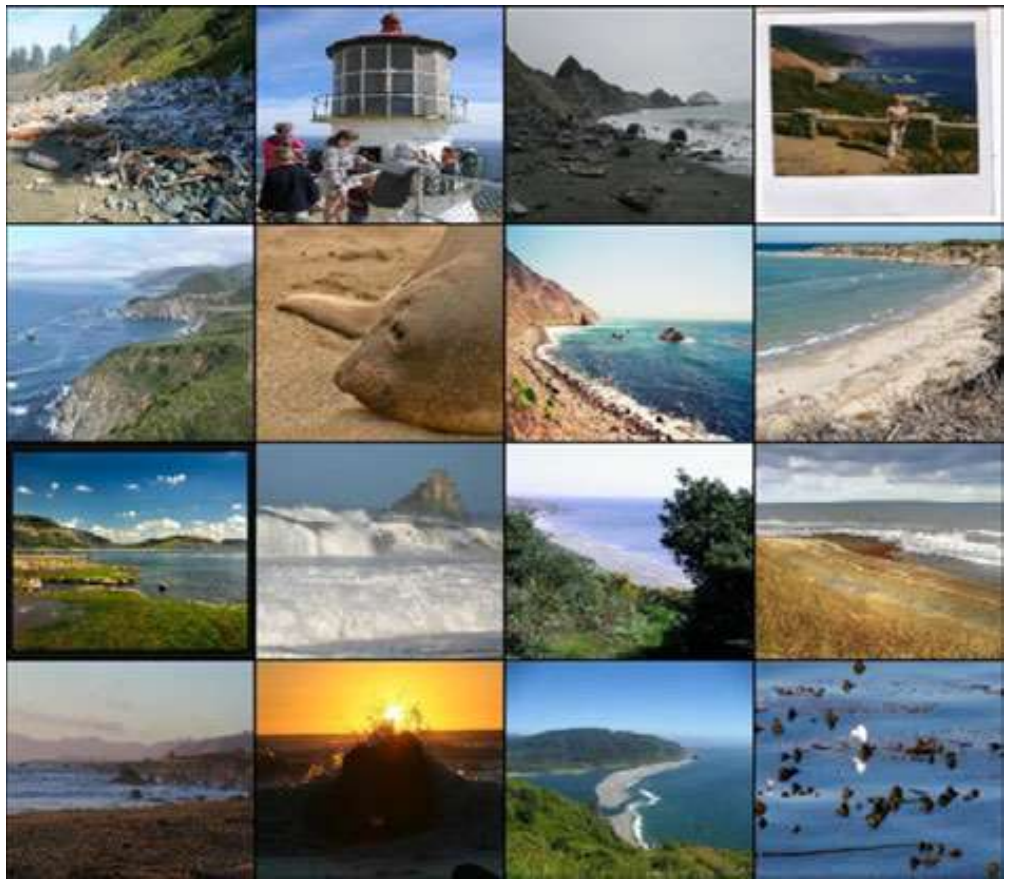


Fig. 17 Top 16

(query-independent) retrieval results for query term sheep

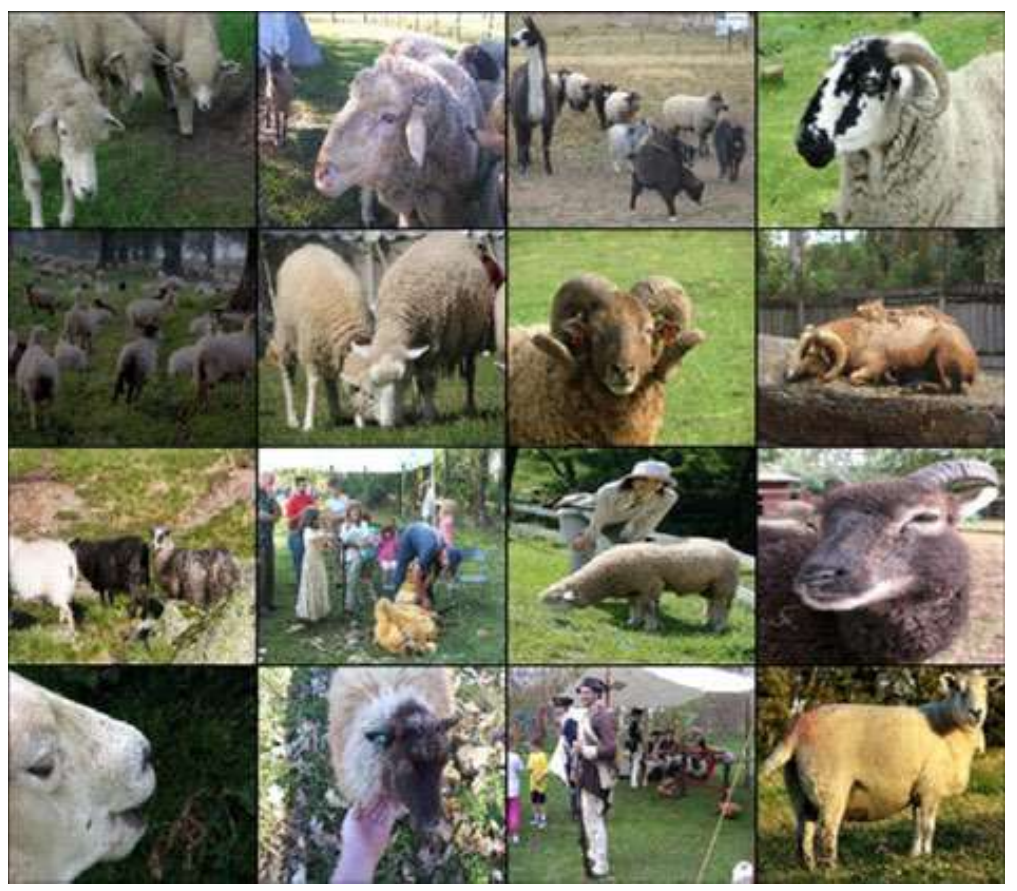

show the top 16 retrieved images for the query terms building and coast. We used the query-dependent approach with $k=250$ neighbors, $\beta=0.2$ and a non-uniform bias setting the top $L=500$ entries. For the query-independent system, Fig. 17 shows the results of using sheep as query term, with $k=500$ neighbors, $\beta=0.2$ and a uniform bias.

\section{Conclusion}

We have presented an image ranking method based on random walk on an image graph. This graph is built from images and their similarities among each other. We proposed to use a multimodal similarity measure to find nearest neighbors of images. Our experiments showed that combining more than one modality improves the system performance significantly. Moreover we proposed a query-independent ranking approach that allows to compute a global ranking score prior to a certain query. In this work we further addressed scalability issues by using a $k$-nearest neighbor approximation when building the graph and computing the similarities between images. Future work will evaluate the query-independent approach on even larger databases, explore its scalability in more detail and evaluate the proposed approach in the context of abstract query terms.

\section{References}

1. Berg TL, Berg AC (2009) Finding iconic images. In: The 2nd internet vision workshop at IEEE CVPR 
2. Crandall D, Backstrom L, Huttenlocher D, Kleinberg J (2009) Mapping the world's photos. In: Proc. 18th international world wide web conference

3. Dempster A, Laird N, Rubin D (1977) Maximum likelihood from incomplete data via the EM algorithm. J R Stat Soc 39:1-38

4. Grangier D, Bengio S (2008) A discriminative kernel-based approach to rank images from text queries. IEEE Trans Pattern Anal Mach Intell 30(8):1371-1384

5. He X, Ma WY, Zhang H (2003) Imagerank: spectral techniques for structural analysis of image database. In: ICME '03: proceedings of the 2003 international conference on multimedia and expo. IEEE Computer Society, Washington, DC, USA, pp 25-28

6. Hofmann T (2001) Unsupervised learning by probabilistic latent semantic analysis. Mach Learn 42(1-2):177-196

7. Hsu WH, Kennedy LS, Chang SF (2007) Video search reranking through random walk over document-level context graph. In: MULTIMEDIA '07: proceedings of the 15th international conference on multimedia. ACM, New York, NY, USA, pp 971-980

8. Jing Y, Baluja S (2008) Visualrank: applying pagerank to large-scale image search. IEEE Trans Pattern Anal Mach Intell 30(11):1877-1890

9. Kamvar SD, Haveliwala TH, Manning CD, Golub GH (2003) Extrapolation methods for accelerating pagerank computations. In: Proceedings of the twelfth international conference on world wide web. ACM Press, pp 261-270

10. Kennedy LS, Naaman M (2008) Generating diverse and representative image search results for landmarks. In: WWW '08: proceeding of the 17th international conference on world wide web. ACM, New York, NY, USA, pp 297-306

11. Langville AN, Meyer CD (2006) Updating markov chains with an eye on google's pagerank. SIAM J Matrix Anal Appl 27(4):968-987

12. Li X, Snoek CG, Worring M (2008) Learning tag relevance by neighbor voting for social image retrieval. In: MIR '08: proceeding of the 1st ACM international conference on multimedia information retrieval. ACM, New York, NY, USA, pp 180-187

13. Li X, Wu C, Zach C, Lazebnik S, Frahm JM (2008) Modeling and recognition of landmark image collections using iconic scene graphs. In: Forsyth DA, Torr PHS, Zisserman A (eds) ECCV (1). Lecture notes in computer science, vol 5302. Springer, pp 427-440

14. Lienhart R, Slaney M (2007) pLSA on large scale image databases. In: IEEE international conference on acoustics, speech and signal processing 2007 (ICASSP 2007), vol IV, pp 12171220

15. Lienhart R, Romberg S, Hörster E (2009) Multilayer plsa for multimodal image retrieval. In: ACM international conference on image and video retrieval (CIVR)

16. Liu D, Hua XS, Yang L, Wang M, Zhang HJ (2009) Tag ranking. In: 18th international world wide web conference, pp 351-351

17. Lowe DG (2004) Distinctive image features from scale-invariant keypoints. Int J Comput Vis 60(2):91-110

18. Nister D, Stewenius H (2006) Scalable recognition with a vocabulary tree. In: IEEE computer society conference on computer vision and pattern recognition (CVPR), vol 2, pp 2161-2168

19. Page L, Brin S, Motwani R, Winograd T (1998) The pagerank citation ranking: bringing order to the web. Tech. rep., Stanford Digital Library Technologies Project

20. Pan JY, Yang HJ, Faloutsos C, Duygulu P (2004) Gcap: graph-based automatic image captioning. In: Conference on computer vision and pattern recognition workshop, 2004. CVPRW 2004, pp 146-146

21. Parzen E (1962) On estimation of a probability density function and mode. Ann Math Stat 33(3):1065-1076

22. Raguram R, Lazebnik S (2008) Computing iconic summaries of general visual concepts. Computer vision and pattern recognition workshop. CVPRW 2008, vol 0, pp. 1-8

23. Romberg S, Hörster E, Lienhart R (2009) Multimodal plsa on visual features and tags. In: IEEE international conference on multimedia and expo (ICME)

24. Schroff F, Criminisi A, Zisserman A (2007) Harvesting image databases from the web. In: IEEE 11th international conference on computer vision, 2007. ICCV 2007, pp 1-8

25. Wang G, Forsyth D (2008) Object image retrieval by exploiting online knowledge resources. In: IEEE conference on computer vision and pattern recognition, pp 1-8

26. Zheng YT, Zhao M, Song Y, Adam H, Buddemeier U, Bissacco A, Brucher F, Chua TS, Neven H (2009) Tour the world: building a web-scale landmark recognition engine. In: Proc. of ICCV, Miami, Florida, USA 


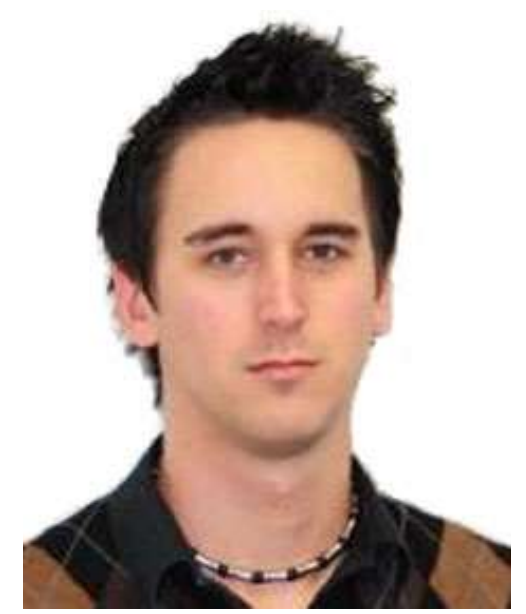

Fabian Richter received his diploma degree in Computer Science from the University of Augsburg, Germany, in November 2009. Since then he works at the Multimedia Computing Lab as a PhD student. His research interests include machine learning, image analysis and image processing.

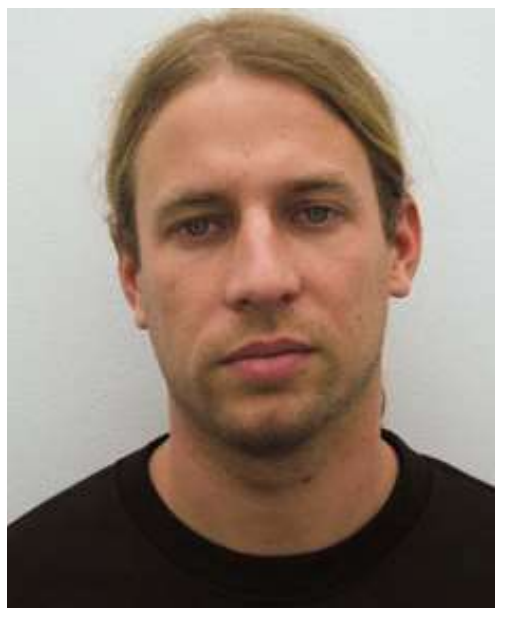

Stefan Romberg Since November 2008, he is a PhD student at the Multimedia Computing Lab of the University of Augsburg, Germany. He received his Diploma Degree in Computer Science from the University of Augsburg, Germany, in November 2008. His research interest include Computer Vison, Multimedia Content Analysis and Image Retrieval. 


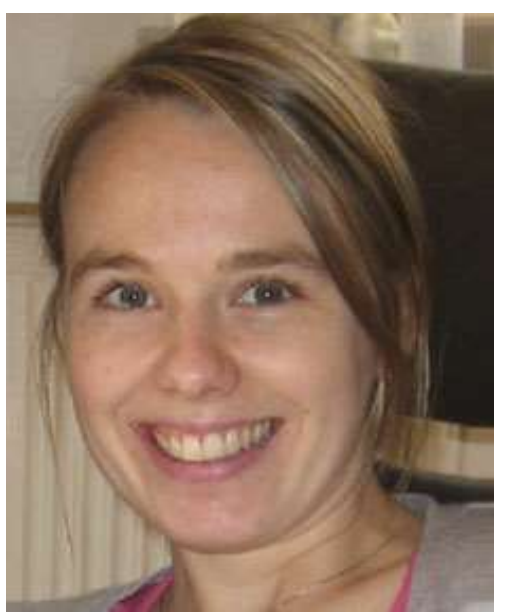

Eva Hörster has her $\mathrm{PhD}$ in Computer Science from the University of Augsburg, Germany in 2009 and received her Diploma Degree in Electrical Engineering from the University of ErlangenNuremberg, Germany, in December 2004. In the spring of 2003 she spent a semester at the Institute National des Science Appliquees, Rennes, France, as a visiting student. She has done an internship at Intel Research, USA in 2004 and one at Yahoo! Research, USA in 2008. Her research interests include Image Retrieval, Multimedia Content Analysis, Machine Learning and Computer Vision.

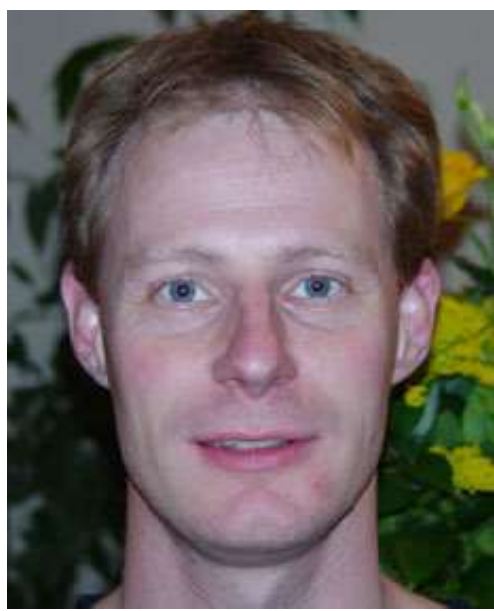

Rainer Lienhart Since August 2004, he is a full professor in the computer science department of the University of Augsburg leading the lab for Multimedia Computing.

From August 1998 to July 2004 he was a Staff Researcher at Intel's Microprocessor Research Lab in Santa Clara, California, where he worked on transforming a network of heterogeneous, distributed computing platforms into an array of audio/video sensors and actuators capable of performing complex DSP tasks such as distributed beamforming, audio rendering, audio/visual tracking, and camera array processing. In particular, this requires putting distributed heterogeneous computing platforms with audio-visual sensors into a common time and space coordinate system. At the same time, he was also continuing his work on media mining, where he is well-known for his work in video content analysis with contributions in text detection/recognition, commercial detection, face detection, shot and scene detection, and automatic video abstraction. 
He received his $\mathrm{PhD}$ in Computer Science from the University of Mannheim, Germany, in 1998, where he was a member of the Movie Content Analysis Project (MoCA).

$\mathrm{He}$ as been a committee member of ACM Multimedia, IEEE International Conference on Multimedia Systems, IEEE International Conference on Multimedia Expo, SPIE Storage and Retrieval of Media Databases, IEEE Workshop on Content-Based Access of Image and Video libraries and the International Eurographics Workshop on Multimedia. He is a reviewer for IEEE Transactions on Pattern Recognition and Machine Learning, IEEE Transaction on Multimedia, Journal of Computer Vision and Image Understanding and ACM Multimedia Systems Journal.

Dr. Lienhart has published over 50+ papers in major conferences and journals and filed $20+$ patents. 